\title{
3
}

\section{Anforderungen von Nutzern flexibler öffentlicher Mobilitätskonzepte an digitale Fahrgastinformationen mit Echtzeitdaten}

K. Viergutz, F. Brinkmann (Deutsches Zentrum für Luft- und Raumfahrt e.V.)

Anforderungen von Nutzern flexibler öffentlicher Mobilitätskonzepte an digitale Fahrgastinformationen mit Echtzeitdaten

Abstract 332

3.1 Ziel und methodisches Vorgehen 332

3.2 Charakterisierung bedarfsorientierter Mobilitätskonzepte 334

3.3 Dynamische Fahrgastinformationen mit Echtzeitdaten im Nahverkehr 334

3.4 Studie zu Nutzungsgewohnheiten von Fahrgastinformationen im Linienverkehr..

3.5 Ableitung neuer Anforderungen an mobile Informationsmedien ....... 341

3.6 Ableitung neuer Anforderungen an stationäre Informationsmedien. 343

3.7 Zusammenfassung der Ergebnisse und Beschreibung von Zukunftsszenarien 345

Literatur 345 


\section{Abstract}

Flexible öffentliche Mobilitätskonzepte mit Eigenschaften wie der Haustürbedienung und digitaler Vernetzung mit Live-Tracking, die sich flexibel an den individuellen Mobilitätsbedarf von Fahrgästen anpassen, gewinnen zusehends an Bedeutung. Daraus ergeben sich neue Herausforderungen an Informationen über verfügbare Mobilitätsangebote. Der Informationsbedarf für die Nutzung des bedarfsorientierten ÖPNV unterscheidet sich von jenem Informationsbedarf zur Nutzung von auf Linien und Fahrplänen basierenden öffentlichen Verkehrsmitteln. Zur Analyse dieser neuen Anforderungen an Fahrgastinformationen werden in diesem Beitrag die Ergebnisse einer Studie des Instituts für Verkehrssystemtechnik des Deutschen Zentrums für Luft- und Raumfahrt e.V. (DLR) präsentiert. Die Studie mit 1.354 Teilnehmern hatte zum Ziel, Erkenntnisse über die Informationsbeschaffung von Fahrgästen im fahrplanbasierten ÖPNV sowie deren Anforderungen an dynamische Fahrgastinformationen mit Echtzeitdaten zu gewinnen. Dabei beschäftigte sich die Befragung insbesondere mit Anzeigen an Haltestellen und mobilen Anwendungen (Smartphone-Apps). Die Ergebnisse dieser Studie gehen ein in die Erforschung innovativer Mobilitätskonzepte: Bei der Frage, wie Fahrgäste sich bei der Nutzung eines haltestellenlosen Nahverkehrssystems über den aktuellen Betriebsablauf informieren würden, spielen insbesondere die Erkenntnisse über die Nutzung mobiler Anwendungen eine wichtige Rolle. Für den vorliegenden Beitrag werden die gewonnenen Erkenntnisse aus der Anforderungsanalyse speziell auf flexible Mobilitätskonzepte der Städte von morgen übertragen, wodurch das Bild zukünftiger Nutzer und ihrer Anforderungen illustriert werden kann.

\subsection{Ziel und methodisches Vorgehen}

Heutzutage besitzen Verkehrsteilnehmer hohe Ansprüche an die Individualisierung ihrer Mobilität (Winterhoff et al, 2009). Mobilitätsangebote, die sich flexibel an den individuellen Mobilitätsbedarf anpassen, gewinnen zusehends an Bedeutung (Mulley \& Nelson 2009; Kleinhückelkotten \& Neitzke 2011). Zudem präferieren Verkehrsteilnehmer Angebote, die sich spontan in ihren Alltag integrieren lassen. Dies zeigt sich an der steigenden Verfügbarkeit bedarfsorientierter Mobilitätsangebote mit Eigenschaften wie der Haustürbedienung und digitalem Live-Tracking. Insbesondere in ländlich geprägten Gemeinden sind diese flexiblen Bedienformen seit einigen Jahrzehnten etabliert (König \& Viergutz 2017). Dabei leisten diese bedarfsgesteuerten Bedienformen als Mischform aus öffentlichem und Individualverkehr (Laws 2009) nicht nur einen Beitrag zur Daseinsvorsorge, sondern 
besitzen zudem das Potenzial, insbesondere während Zeiten geringerer Verkehrsnachfrage zur Kostendeckung und damit zur langfristigen Erhaltung des Verkehrsangebots beizutragen (Dalkmann \& Ötting 2004; Enoch, Potter, Parkhurst, \& Smith 2004). Dieser Effekt reduziert gleichzeitig die Schadstoffemission der Fahrzeuge (Iacometti, Setti, Scholliers, Gorini \& Eloranta 2003). Sowohl in urbanen wie auch in dispersen Räumen ist ein Trend zu bedarfsorientierten Mobilitätsangeboten erkennbar.

Große Veränderungen technologischer Mittel ermöglichen neue Ansätze der Gestaltung von Mobilitätskonzepten (Schnieder 2014; Mulley \& Nelson 2009). Fortschreitende Digitalisierung sowie die Allgegenwart mobiler Geräte, wie zum Beispiel Smartphones und Tabletcomputer, stellen als Enabler die technischen Mittel zur Verfügung, die neuartige Möglichkeiten bewirken. Der vorliegende Beitrag versucht Antworten auf zentrale Fragen hinsichtlich der Änderungen der Anforderungen an digitale Fahrgastinformationen bei bedarfsorientierten Bedienformen im Nahverkehr zu geben. Dabei wird aufgezeigt, inwiefern sich die Mobilitätsbedürfnisse der Zukunft ändern. Zudem findet eine Beschreibung der Auswirkungen dieser Entwicklung auf digitale Fahrgastinformationen statt.

\section{Abbildung 3.1 Methodisches Vorgehen der Untersuchung}

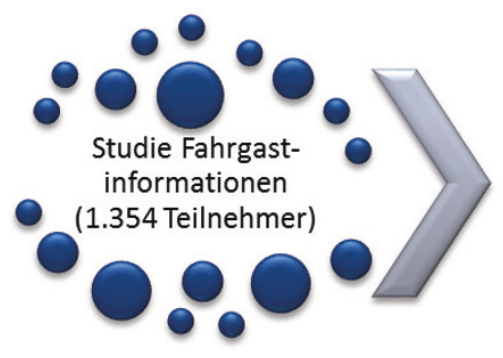

Ableitung von Anforderungen
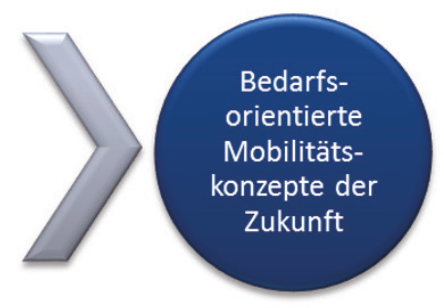

Zur Nutzung des bedarfsorientierten ÖPNV sind andere Informationen erforderlich als zur Nutzung von auf Linien und Fahrplänen basierenden öffentlichen Verkehrsmitteln. Zur Analyse dieser neuen Anforderungen an Fahrgastinformationen werden in diesem Beitrag die Ergebnisse einer Studie mit 1.354 Teilnehmern präsentiert, die zum Ziel hatte, Erkenntnisse über die Informationsbeschaffung von Fahrgästen im fahrplanbasierten ÖPNV sowie deren Anforderungen an dynamische Fahrgastinformationen mit Echtzeitdaten zu gewinnen. Dabei beschäftigte sich die Befragung insbesondere mit Anzeigen an Haltestellen und mobilen An- 
wendungen (Smartphone-Apps). Auf Basis der durch die Studie gewonnenen Erkenntnisse werden Anforderungen von Nutzern flexibler Mobilitätskonzepte abgeleitet (Abbildung 3.1).

\subsection{Charakterisierung bedarfsorientierter Mobilitätskonzepte}

Konventionelle öffentliche Verkehrssysteme besitzen überwiegend ex-ante aufgestellte Fahrpläne. Diese Soll-Fahrpläne geben an, zu welcher Zeit die im Verkehrsnetz entlang definierter Linienwege verorteten Haltestellen bedient werden. Um das Verkehrsangebot auf eine variable Nachfrage anzupassen, kann von diesen festgelegten Haltestellen, Linienwege und Bedienzeiten abgewichen werden, sodass bedarfsorientierte Verkehrsangebote (engl. demand responsive transport) entstehen (Atasoy, Ikeda \& Ben-Akiva 2014). Hierbei sind vielfältige Grade der Flexibilisierung des Angebots möglich. Eine sehr einfache Form der Flexibilisierung ermöglicht der Bedarfslinienverkehr, wie zum Beispiel Anruf-Sammel-Taxis (Bundesministerium für Verkehr, Bau und Stadtentwicklung 2009). Die größte Flexibilität ermöglicht der Bedarfsverkehr im Flächenbetrieb (Mehlert 2001). Nutzer dieses Verkehrssystems können Start- und Zielpunkte ihrer Reise sowie die gewünschte Abfahrts- oder Ankunftszeit innerhalb eines bestimmten Bediengebiets und innerhalb bestimmter Bedienzeiten weitestgehend selbst bestimmen. Für die Fahrgäste resultiert durch die Tür-zu-Tür-Merkmale eines Bedarfsbusses ein höherer Komfort im Sinne einer Mobility on Demand. Damit wird den Anforderungen von Fahrgästen nach stärkerer Individualisierung ihrer Mobilität Rechnung getragen (Bültemann, Viergutz \& Scheier 2016). Die Integration des Mobility on Demand Systems (MODS) als Zu- oder Abbringer zu einem auf Bündelungseffekte ausgerichteten angebotsorientiert bedienten Verkehrssystem berücksichtigt das Bedürfnis der Fahrgäste nach einer zuverlässigen Realisierung von über das Quartier hinausgehenden Mobilitätsbedarfen. Bedarfsorientierte Systeme stellen damit eine flächenhafte Ergänzung zum Linienverkehr mit präformierten Fahrplänen dar (Dalkmann \& Ötting 2004).

\subsection{Dynamische Fahrgastinformationen mit Echtzeitdaten im Nahverkehr}

Fahrgastinformationen erlauben Fahrgästen die Informationsgewinnung über Abfahrten und Verbindungen vor, während und nach der Durchführung einer Reise 
(Scheier 2013). Dabei werden Solldaten, die festgelegte Fahrplandaten widerspiegeln sowie Echtzeitdaten, die Auskunft über die aktuelle Fahrplanlage geben, unterschieden. Echtzeiten werden oft in Form von Restzeiten ausgegeben. Ihre Darstellung erfolgt demnach nicht als absolute Uhrzeiten, sondern als "Countdown" bis zur Abfahrt (Viergutz 2016). Die Sortierreihenfolge entspricht dabei meist der chronologischen Reihenfolge ab dem aktuellen Zeitpunkt. Angezeigte Uhrzeiten hingegen geben in der Regel die Fahrplansolldaten an (Gängrich 2015; Zweckverband Verkehrsverbund Bremen/ Niedersachsen 2013). So kann bei Verspätungen oder fehlenden Standortsignalen der Fahrzeuge eine Kombination aus Echtzeitund Fahrplansolldaten vermittelt werden. Weit verbreitet ist die Restzeitanzeige in ganzen Minuten, eine sekundengenaue Angabe ist jedoch auch möglich (Abbildung 3.2). Echtzeitangaben können auch in Form von Entfernungsangaben des Fahrzeugs zur betreffenden Haltestelle angegeben werden. Aus der Information über die Anzahl der Haltestellen, die zwischen dem Fahrgast und dem Fahrzeug liegen, kann der Fahrgast die prognostizierte Dauer bis zum Eintreffen des Fahrzeugs ableiten. Neben bahnsteig- und gleisspezifischen Anzeigen können Abfahrtstafeln auch die Abfahrten mehrerer Gleise, beispielsweise an Knotenpunkten, enthalten. Solche Abfahrtstafeln, welche sich nicht direkt am Bahnsteig, jedoch in dessen Nähe befinden, werden auch "Near-Stop-Displays“ genannt (Viergutz 2016). Insgesamt tragen digitale Fahrgastinformationen zur durch den Nutzer wahrgenommenen Zuverlässigkeit des Gesamtsystems Nahverkehr bei (Dziekan \& Kottenhoff 2006). 
Abbildung 3.2 Beispiele verschiedener Anzeigeformate: Echtzeitdaten werden meist als Countdown angezeigt, Solldaten in Form von Uhrzeiten.

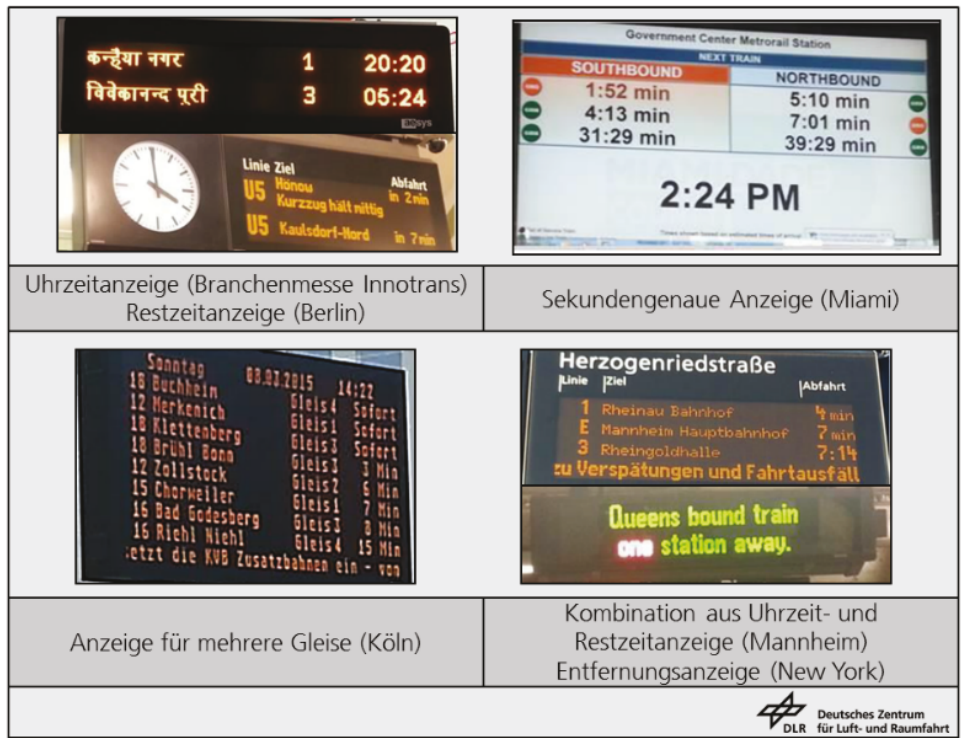

\subsection{Studie zu Nutzungsgewohnheiten von Fahrgastinformationen im Linienverkehr}

Abbildung 3.3 Demografische Angaben der Studienteilnehmer.

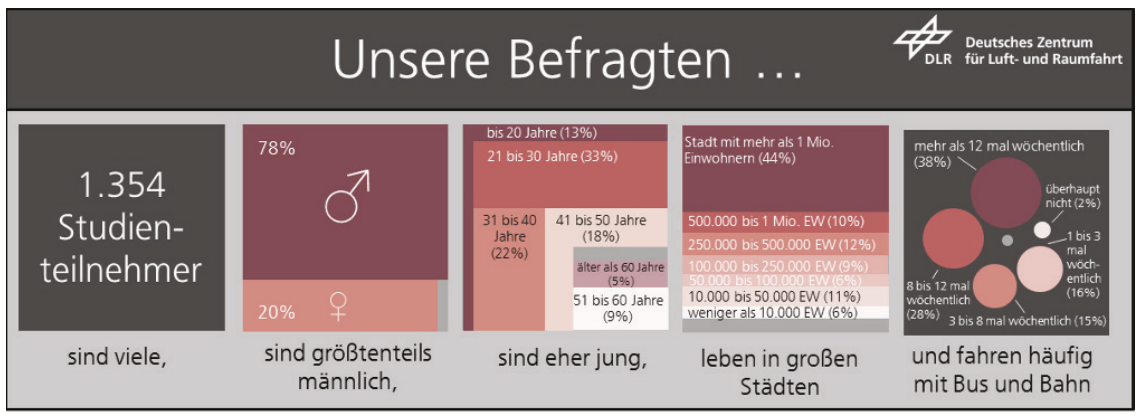


Die Online-Befragung wurde im Untersuchungszeitraum vom 24.11.2016 bis 11.12.2016 unter 1.354 Studienteilnehmern durchgeführt. Rund 78 Prozent davon sind männlichen Geschlechts. Zudem ist ein Großteil der Befragten (68 Prozent) unter 41 Jahren alt. Insgesamt leben die Befragten eher in großen Städten und nutzen häufig öffentliche Verkehrsmittel (Abbildung 3.3).

\section{Abbildung 3.4 Informationsbeschaffung im ÖPNV: Mobile Anwendungen, Stadtplanauskünfte und Suchanfragen per Webseiten führen die Befragten zum Ziel.}

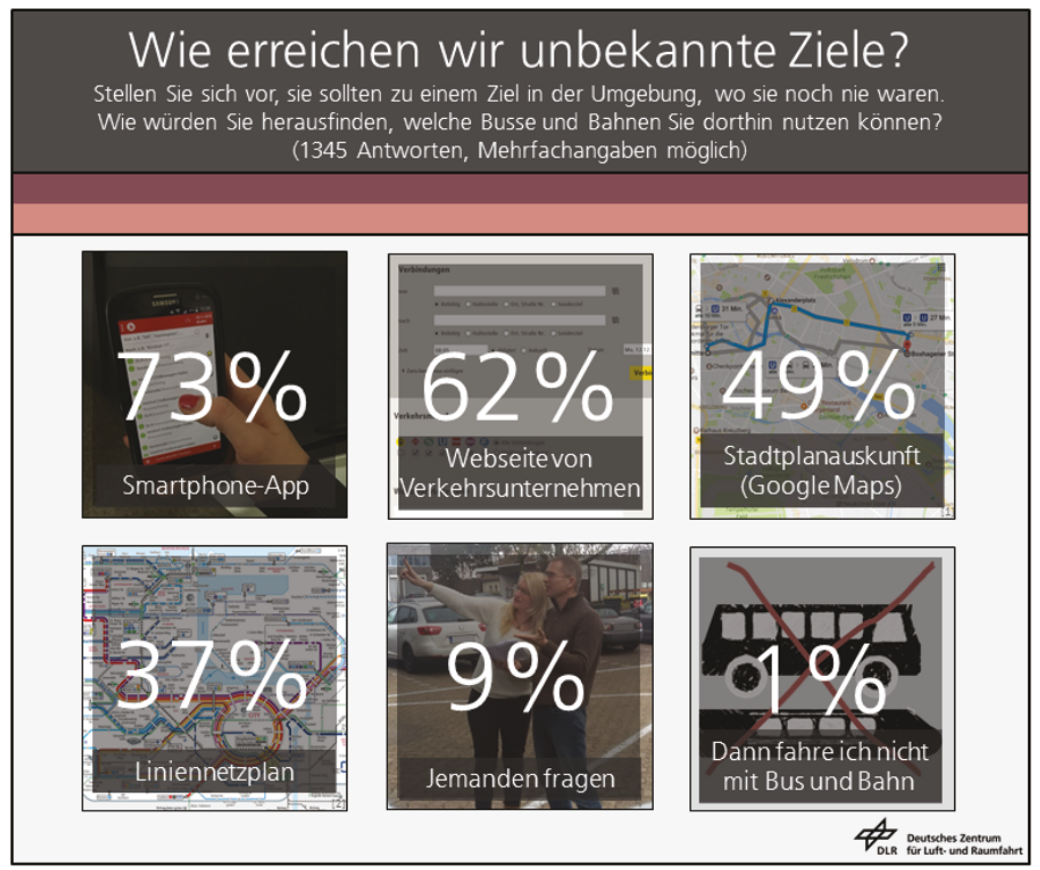

In der Studie wurden Anwendungsfälle verschiedener Auskunftsmedien beim Wayfinding im Nahverkehr untersucht. Die Befragten sollten anhand eines Szenarios entscheiden, wie sie sich Informationen zur Erreichung eines ihnen unbekannten Zieles in der Umgebung beschaffen würden (Abbildung 3.4). Dabei waren Mehrfachangaben möglich. Rund 73 Prozent der Befragten würden eine mobile Anwendung zur Informationsgewinnung nutzen. Webseiten eines Verkehrsunternehmens, die mithilfe eines mobilen oder stationären Ausgabegerätes aufgerufen werden, würden rund 62 Prozent zurate ziehen. Rund 49 Prozent würden sich 
durch eine dynamische Onlinekarte (Stadtplanauskunft) unterstützen lassen. Außerdem sind die eigenständige, nicht technikgestützte Generierung von Verbindungen anhand eines Liniennetzplanes (37 Prozent) sowie die Befragung einer anderen Person (z.B. Bekanntenkreis, Mitarbeiter des Verkehrsunternehmens, neun Prozent) häufig gewählte Möglichkeiten zur Informationsbeschaffung. Bei der Differenzierung der Antworten nach Altersklassen wird deutlich, dass Apps und Webseiten in allen Altersklassen beliebte Auskunftsmedien darstellen. Dabei nutzen Befragte bis zum Alter von 40 Jahren etwas häufiger Apps zur Informationsgewinnung, wohingegen Befragte ab einem Alter von 41 Jahren etwas häufiger Webseiten nutzen. Auch bei der Aufschlüsselung der Antworten nach Geschlechtern zeigt sich, dass sowohl männliche wie auch weibliche Befragte vor allem die Nutzung mobiler Anwendungen sowie von Webseiten von Verkehrsunternehmen angaben. Dabei ist die Nutzung mobiler Anwendungen bei männlichen, die Nutzung von Webseiten dagegen bei weiblichen Studienteilnehmern etwas stärker ausgeprägt.

Abbildung 3.5 Nutzungshäufigkeit von mobilen Anwendungen und öffentlichen Verkehrsmitteln: Wer häufig den ÖPNV nutzt, nutzt auch häufig Smartphone-Apps dafür.

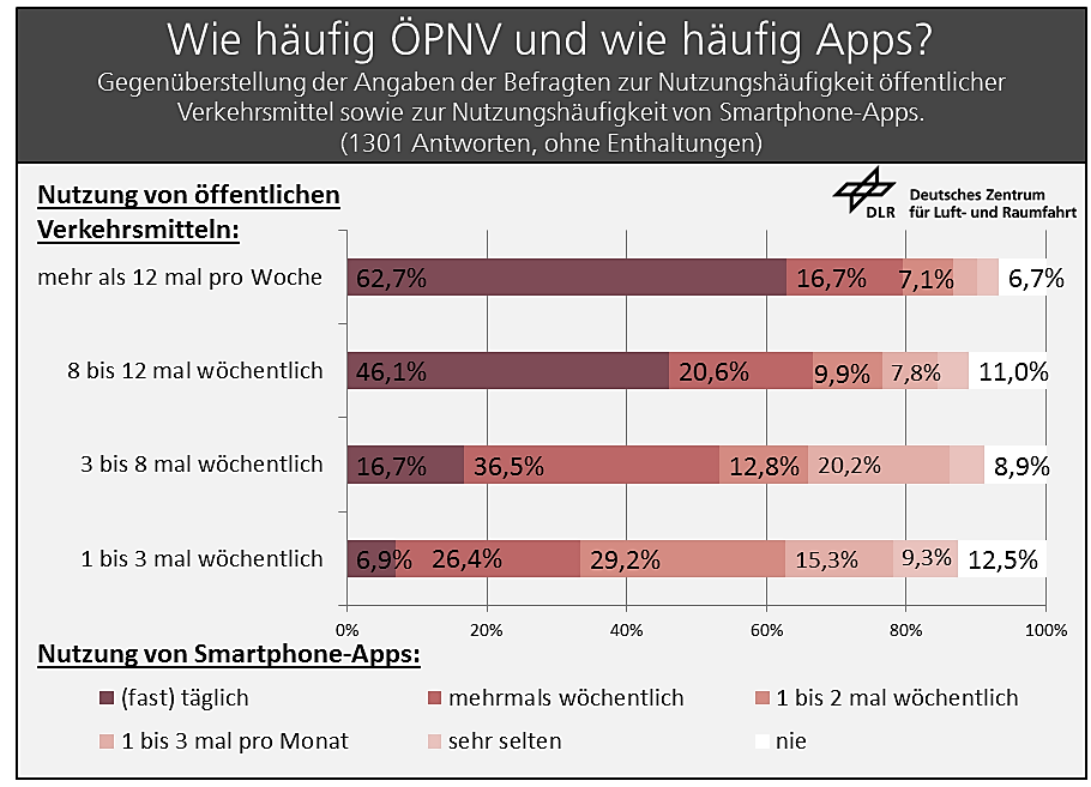


Mobile Anwendungen für öffentliche Verkehrsmittel werden von den Studienteilnehmern im Alltag für gewöhnlich häufig genutzt: Ein Anteil von 40,3 Prozent der Befragten gab an, täglich Apps zu nutzen, 21,9 Prozent mehrmals wöchentlich.

Die vorangegangene Frage hat ergeben, dass auf unbekannten Verbindungen insbesondere mobile Anwendungen sowie Webseiten zur Auskunft genutzt werden. Diese Erkenntnis legt den Verdacht nahe, dass mobile Anwendungen insbesondere dort als nutzenstiftend empfunden werden, wo eine geringe System- und Ortskenntnis seitens des Nutzers vorherrscht. Die Kombination der Nutzungshäufigkeit von öffentlichen Verkehrsmitteln mit der Nutzungshäufigkeit von Apps zeigt jedoch, dass 62,7 Prozent der Befragten, die angaben, mehr als zwölfmal wöchentlich öffentliche Verkehrsmittel zu nutzen, (fast) täglich Apps zur ÖPNV-Auskunft nutzen (Abbildung 3.5). Insgesamt korreliert die Nutzungshäufigkeit von öffentlichen Verkehrsmitteln also mit der Nutzungshäufigkeit von Apps. Dieses Ergebnis zeigt, dass mobile Anwendungen nicht nur in unbekannter Umgebung eingesetzt werden, sondern gerade dort Nutzen stiften, wo Anwender bereits über System- und Ortskenntnis verfügen. Ein Erklärungsansatz für dieses Studienergebnis könnte sein, dass Nutzer bei der Informationsgewinnung ein gewohnheitsmäßiges Verhalten zeigen und bewährte Informationsquellen immer wieder zurate ziehen (Naab 2013).

Mobile Anwendungen erlauben zwei Arten von Abfragen: Bei der Abfahrtsauskunft werden aktuelle Abfahrten an ausgewählten Haltestellen und Linien abgefragt. Bei der Verbindungsabfrage werden Auskünfte von einem beliebigen Startzu einem beliebigen Zielpunkt erteilt, wodurch eine Reiseplanung im Voraus möglich ist. Die Studie hat ergeben, dass 30,3 Prozent der Befragten hauptsächlich Verbindungsauskünfte in Apps nutzen. Für 45,4 Prozent sind beide Auskunftsarten gleich wichtig (Abbildung 3.6). 


\section{Abbildung 3.6 Bevorzugte Auskunftsarten in mobilen Anwendungen: Verbin- dungsauskünfte werden häufiger genutzt als Abfahrtsaus- künfte - beide Auskunftsarten ergänzen sich jedoch.}

\section{Von hier weg oder von $A$ nach $B$ ?}

Welche dieser Auskunftsarten nutzen Sie hauptsächlich in Apps? (1339Antworten)

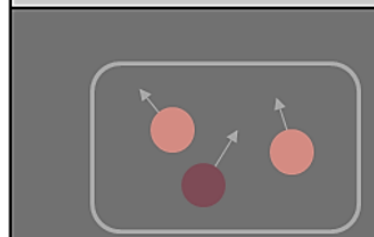

Abfahrtsauskunft $13,4 \%$

Abfahrten an einer Haltestelle

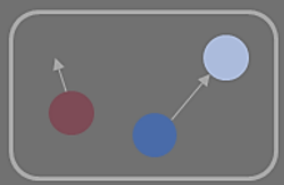

Beides ist gleich wichtig $45,4 \%$

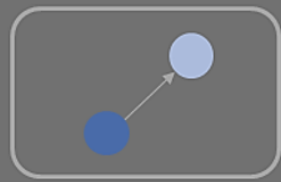

Verbindungsauskunft $30,3 \%$

Neben Anforderungen an mobile Anwendungen wurden die Studienteilnehmer auch um Feedback bezüglich Haltestellenanzeigen gebeten. Bei Haltestellenanzeigen handelt es sich um stationäre Informationssysteme, die eine kollektive Nutzerschaft bedienen. Die Befragung ergab, dass rund 84,5 Prozent der Befragten der Aussage „[...] Diese Anzeigen halte ich für sinnvoll und nützlich“ voll und ganz zustimmten. Weitere 12,2 Prozent der Befragten gaben an, dieser Aussage eher zuzustimmen.

Ein besonders prägnantes Ergebnis der Befragung ist die gleich hoch eingeschätzte Wichtigkeit der beiden Informationssysteme Apps und Haltestellenanzeigen. Für 40,1 Prozent der Befragten stellen Anzeigen an Haltestellen die wichtigste Fahrgastinformation dar. Nicht auf Auskünfte per mobiler Anwendung verzichten möchten 40,2 Prozent der Befragten (Abbildung 3.7). 
Abbildung 3.7 Fazit: Wichtigste Fahrgastinformation aus Fahrgastsicht - die Kombination ist wichtig.

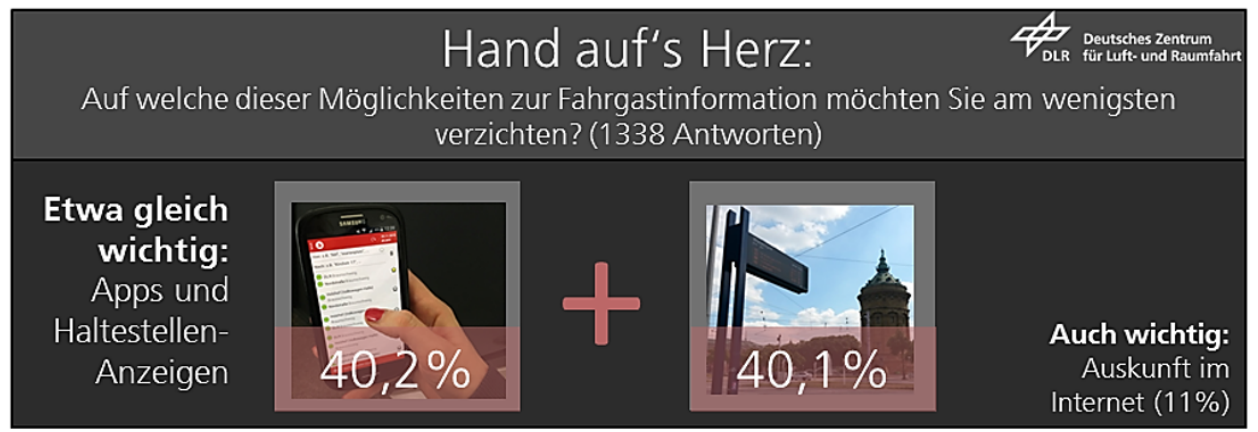

Die Studie ergab, dass Haltestellenanzeigen trotz der ständigen Verfügbarkeit von Smartphones immer noch wichtige Informationsmedien im ÖPNV sind und dass Apps und Haltestellenanzeigen nicht alleine ausreichen sondern sich gegenseitig ergänzen. Daraus resultieren neue Anforderungen an haltestellenlose Nahverkehrssysteme.

\subsection{Ableitung neuer Anforderungen an mobile Informationsmedien}

Im Folgenden werden auf Basis der Erkenntnisse aus der beschriebenen Studie Anforderungen an mobile Informationsmedien zur Nutzung bedarfsorientierter Mobilitätskonzepte abgeleitet.

Die Vielfalt von Fahrgastinformationen wird wertgeschätzt.

Unterschiedliche Fahrgastinformationen bieten unterschiedliche Funktionen. Den größten Nutzen stiftet eine sinnvolle Kombination kollektiver und individueller Ausgabemedien, welche entsprechend dem aktuellen Informationsbedarf eingesetzt werden.

Bei fehlender Orts- und Systemkenntnis bieten insbesondere mobile Anwendungen Unterstützung. 
Mobile Anwendungen sollen sich weiterentwickeln von der reinen Verbindungsauskunft hin zum individualisierbaren, intelligenten und echtzeitbasierten Informationsmedium. Das Smartphone wird zum Reiseassistenten.

Mobile Anwendungen werden häufig zur Informationsbeschaffung im Nahverkehr genutzt.

Fahrgäste sind geübt in der Nutzung mobiler Anwendungen. Dies kann darauf hindeuten, dass erweiterte Funktionalitäten mobiler Anwendungen nutzenstiftend sein könnten.

Ortung auf dem Stadtplan und Umgebungsinformationen können in mobilen Anwendungen zusätzlichen Nutzen stiften.

Zur erweiterten Funktionalität mobiler Anwendungen könnte die Umgebungsinformation zählen. Dabei erhält der Nutzer beispielsweise Informationen über Points of Interest, Einkaufsmöglichkeiten, die aktuelle Verkehrslage oder Veranstaltungen in seiner Umgebung.

Befragte, die häufig Nahverkehrsmittel nutzen, nutzen auch häufig mobile Anwendungen dafür.

Mobile Anwendungen stiften nicht nur in unbekannter Umgebung Nutzen, sondern dienen ebenso der Information über den aktuellen Betriebsablauf auf gewohnten Verbindungen. Denkbar wäre also die Erweiterung bisheriger Funktionalitäten mobiler Anwendungen um Informationen, die insbesondere für Nutzer mit guter System- und Ortskenntnis von Interesse sein könnten.

Mithilfe mobiler Anwendungen werden bevorzugt Verbindungsauskünfte abgefragt. Echtzeitdaten und Informationen über die aktuelle Betriebslage werden als wichtig empfunden.

Individualisierte und aktuelle Auskünfte sind Fahrgästen wichtig. Auch zur Befriedigung dieses Bedürfnisses kann die Weiterentwicklung mobiler Anwendungen zu Reiseassistenten beitragen. 


\subsection{Ableitung neuer Anforderungen an stationäre Informationsmedien}

Die Auswertung der Studie hat ergeben, dass stationäre Informationsmedien (trotz mobiler Anwendungen) derzeitig unverzichtbare Funktionen in bestehenden Nahverkehrskonzepten erfüllen. Da die beschriebenen bedarfsorientierten Mobilitätskonzepte keine festgelegten und gekennzeichneten Haltestellen besitzen, sondern den Zustieg auch abseits markierter Haltestellen ermöglichen, bietet sich hierbei keine Möglichkeit zur Einrichtung von Haltestellenanzeigen. Zur Befriedigung dieses bestehenden Informationsbedarfs, der aus Nutzersicht nicht allein durch mobile Auskunftsmedien abgedeckt werden kann, muss deshalb ein Äquivalent gefunden werden, welches die Aufgabe der Informationsbereitstellung am Abfahrtsort übernimmt, wodurch keine Nutzeraktivität erforderlich ist sondern gebotene Informationen lediglich konsumiert werden können. Hierdurch könnte auch dem Problem begegnet werden, dass nicht jeder Nutzer über ein mobiles Gerät verfügt.

Hierfür eignen sich Informationsmedien im öffentlichen Raum, die kollektiv nutzbar sind und den Abruf echtzeitbasierter und individualisierter Auskünfte ermöglichen. Eine Möglichkeit zur Bereitstellung dieser stationären Informationsmedien sind sogenannte Public Displays, die in manchen Linienverkehrssystemen bereits existieren.

Public Displays sind stationäre Bildschirme im öffentlichen Raum (Viergutz 2016). Dabei handelt es sich um kollektive Auskunftsmedien, welche die nächsten Abfahrten öffentlicher Verkehrsmittel an mehreren Haltestellen in der Umgebung präsentieren. Individuelle Auskünfte über Abfahrten zu einem späteren Zeitpunkt oder an anderen Orten sind nicht möglich. Abbildung 3.8 zeigt Beispiele solcher Public Displays im Linienverkehr. 


\section{Abbildung 3.8 Beispielhafte Public Displays im Linienverkehr.}

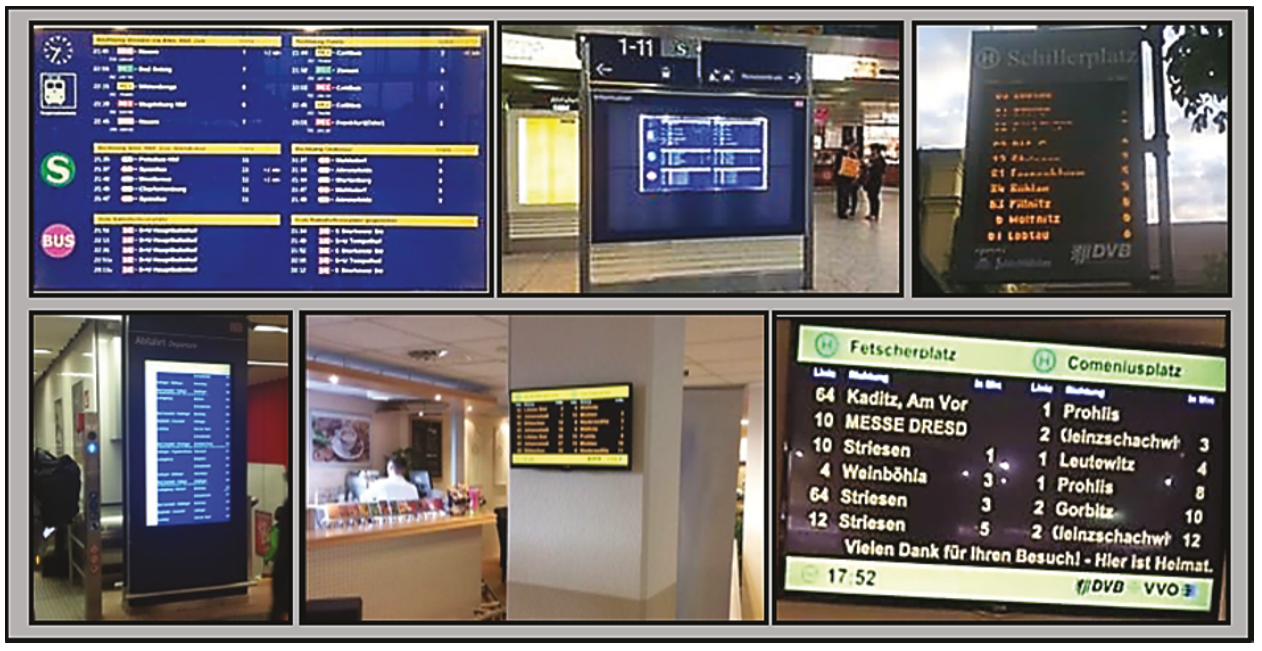

Die 1.354 Teilnehmer der beschriebenen Studie wurden um ihre Einschätzung des Nutzwerts von Public Displays im Linienverkehr gebeten. Dabei stimmten 54 Prozent der Befragten der Aussage „Public Displays halte ich für sinnvoll und nützlich“ voll und ganz zu, weitere 29 Prozent stimmten der Aussage eher zu.

Zur Nutzung bedarfsorientierter Mobilitätskonzepte kann eine Erweiterung der Funktionalität dieser Public Displays die bisher nutzenstiftenden Haltestellenanzeigen ersetzen. So könnten sich Public Displays zu Buchungsterminals weiterentwickeln. Eine echtzeitbasierte Auskunft über die aktuelle Betriebslage sowie die Möglichkeit zur Buchung von Fahrten, beispielsweise anhand eines Touch-Terminals, würde auch ohne den Einsatz mobiler Anwendungen die uneingeschränkte Nutzung des Verkehrssystems ermöglichen. Dadurch könnten auch stationäre Informationsmedien die identifizierten Anforderungen individualisierter Fahrgastinformationen erfüllen. Stationäre Informationsmedien würden durch diese Ergänzung der bereits vorhandenen kollektiven Information durch die Möglichkeit zur Interaktion zwischen Nutzer und Informationsmedium zudem auch individuelle Informationsbedürfnisse erfüllen. 


\subsection{Zusammenfassung der Ergebnisse und Beschreibung von Zukunftsszenarien}

Die in diesem Beitrag vorgestellte Studie ergab unter anderem, dass sowohl mobile wie auch stationäre Informationsmedien von Fahrgästen geschätzt werden. Durch die unterschiedlichen Funktionen und Wege der Informationsgewinnung erfüllen mobile und stationäre Informationsmedien unterschiedliche Nutzungszwecke und ergänzen sich zu einem ganzheitlichen und komfortablen Informationsbündel.

Bedarfsorientierte Mobilitätskonzepte sehen eine stärkere Individualisierung von Fahrtwünschen vor. Aus dieser erweiterten Funktion von Verkehrssystemen ergeben sich neue Anforderungen an Informationsmedien, die in diesem Beitrag beschrieben wurden.

Zukünftig wird Fahrgastinformationen eine neue Rolle zukommen. Sie dienen nicht mehr lediglich der Information über Abfahrten und Verbindungen sondern erfüllen die Funktionen eines individualisierbaren Reiseassistenten. Dabei werden Auskünfte, die auf Echtzeitdaten basieren, immer wichtiger (Scheier 2013). Insgesamt werden individuelle Auskünfte gegenüber kollektiven Informationen ein immer höheres Gewicht bekommen (Schnieder 2014).

\section{Literatur}

[1] Atasoy, B.; Ikeda, T.; Ben-Akiva, M. (2014): The Concept and Impact Analysis of a Flexible Mobility on Demand System

[2] Bundesministerium für Verkehr, Bau und Stadtentwicklung (2009): Handbuch zur Planung flexibler Bedienungsformen im ÖPNV. Ein Beitrag zur Sicherung der Daseinsvorsorge in nachfrageschwachen Räumen. Abrufbar unter: http://www.bbsr.bund.de/BBSR/DE/Veroeffentlichungen/BMVBS/Sonderveroeffentlichungen/2009/DL_HandbuchPlanungNeu.pdf?_blob=publicationFile\&v=2

[3] Bültemann, S.; Viergutz, K.; Scheier, B. (2017): Differenzierte Bedienung im ÖPNV: Wirtschaftlichkeitsanalyse von bedarfsorientierten Bedienkonzepten im städtischen Busverkehr. Der Nah-verkehr, Ausgabe 1-2017, S. 35-37

[4] Dalkmann, H., Ötting, T. (2004): Flexible Angebotsformen - Möglichkeiten zur Kosteneinsparung bei verbessertem Angebot? Erschienen in: Kagermeier, A. (Hrsg.) Verkehrssystem- und Mobilitätsmanagement im ländlichen Raum; Studien zur Mobilitäts- und Verkehrsforschung, Band 10. S. 75-90

[5] Dziekan, K.; Kottenhoff, K. (2006): Dynamic at-stop real-time information displays for public transport: effects on customers. Transportation Research Part A 41 (2007) S. 489501

[6] Enoch, M.; Potter, S.; Parkhurst, G.; Smith, M. (2004): Indermode: innovations in De- 
mand Responsive Transport. Department for Transport and Greater Manchester Passenger Transport Executive. Final report. London. Abrufbar unter: www.dft.gov.uk

[7] Gängrich, P. (2015): Echtzeit und „HOCHBAHN-Minute“ - was steckt hinter FIMS? Abrufbar unter: http://dialog.hochbahn.de/bus-in-zukunft/echtzeit-und-hochbahn-minutewas-steckt-hinter-fims/

[8] Iacometti, A.; Setti, L.; Scholliers, J.; Gorini, M.; Eloranta, P. (2003): Technologies for DRT Systems. Erschienen in: Ambrosino, G.; Nelson, J.; Romanazzo, M. (Hrsg): Demand Responsive Transport Services: Towards the Flexible Mobility Agency, ENEA, Italian National Agency for New Technologies, Energy and the Environment.

[9] Kleinhückelkotten, S.; Neitzke, H. (2011): Lebensstile der Zukunft, erschienen in: Demuth, B.; Heiland, S.; Wiersbinski, N.; Finck, P.; und Schiller, J. (2011): Landschaften in Deutschland - Der stille Wandel, BNF-Skripten 303, Seiten 49-68

[10] König, A.; Viergutz, K. (2017): Der Fahrschein für den Anrufbus: Tarifgestaltung von bedarfsgesteuerten Bedienformen des öffentlichen Verkehrs im ländlichen Raum, erschienen in: Der Nahverkehr, Ausgabe 6-2017. S. 11-15, Juni 2017

[11] Laws, R. (2009): Evaluating publicly funded DRT schemes in England and Wales, PhD Thesis, Loughborough University, Loughborough

[12] Mehlert, C. (2001): Die Einführung des AnrufBus im ÖPNV. Praxiserfahrungen und Handlungsempfehlungen. Schriftenreihe für Verkehr und Technik, Bd. 91., Bielefeld

[13] Mulley, C.; Nelson, J. (2009): Flexible transport services: A new market opportunity for public transport. Research in Transportation Economics, 25. Aufl., S. 39-45

[14] Naab, T. (2013): Gewohnheiten und Rituale der Fernsehnutzung. Theoretische Konzeption und methodische Perspektiven. Reihe Rezeptionsforschung, Band 27.

[15] Scheier, B. (2013): Entwicklung von Anwendungen zur Qualitätssteigerung im ÖPNV auf Grundlage von Echtzeitdaten - Nutzung des AIM Basisdienst ÖPNV-Daten. Deutsches Zentrum für Luft- und Raumfahrt. Institut für Verkehrssystemtechnik. Braunschweig. Abrufbar unter: http://elib.dlr.de/92357/1/ Masterarbeit_BenediktScheier_DLR_TUBS_ELIB.pdf

[16] Schnieder, K. (2014): Öffentlicher Personennahverkehr im Jahre 2050 - Was könnte wirklich anders sein? Flexibilisierung des Nahverkehrs. Erschienen in: Technikfolgenabschätzung. Theorie und Praxis 23. Jg., Heft 1, S. 38-45, April 2014

[17] Viergutz, K. (2016): Echtzeitdaten im ÖPNV - Welche Anforderungen haben Fahrgäste und was ist besser: Apps oder Haltestellen-Anzeigen? Internationales Verkehrswesen, Ausgabe 4-2016, S. 47-49

[18] Winterhoff, M.; Kahner, C.; Ulrich, C.; Syler, P.; Wenzel, E. (2009): Zukunft der Mobilität 2020 - Die Automobilindustrie im Umbruch?

[19] Zweckverband Verkehrsverbund Bremen/Niedersachsen (2013): Fahrgastinformation im Verkehrsverbund Bremen/Niedersachsen: Qualitätsanforderungen. Abrufbar unter: http://www.zvbn.de/bibliothek/data/Fahrgastinformation-im-VBN.pdf 